\title{
Prediction of Mineralization Prospects Based on Geological Semantic Model and Mobile Computer Machine Learning
}

\author{
Zhengzhen An, ${ }^{1}$ Yue Zhao, ${ }^{1,2}$ Yanfei Zhang, ${ }^{1,3}$ and Xuguang Li $\mathbb{D}^{3}$ \\ ${ }^{1}$ Mining College of Liaoning Technical University, Fuxin, 123000 Liaoning, China \\ ${ }^{2}$ Institute of Innovation and Development for Fuxin Transition, Liaoning Technical University, Fuxin, 123000 Liaoning, China \\ ${ }^{3}$ Shenyang Geological Survey Center, China Geological Survey, Shenyang, 110034 Liaoning, China
}

Correspondence should be addressed to Xuguang Li; lixuguang@mail.cgs.gov.cn

Received 23 April 2021; Revised 6 July 2021; Accepted 6 November 2021; Published 25 November 2021

Academic Editor: Wenqing Wu

Copyright ( ) 2021 Zhengzhen An et al. This is an open access article distributed under the Creative Commons Attribution License, which permits unrestricted use, distribution, and reproduction in any medium, provided the original work is properly cited.

Mineral resources are indispensable in the development of human society and are the foundation of national economic development. As the prospecting target shifts from outcrop ore to concealed ore, from shallow to deep, the difficulty of prospecting becomes more and more difficult. Therefore, the prediction of mineralization prospects is of great significance. This paper is aimed at completing the prediction of mineralization prospects by constructing geological semantic models and using mobile computer learning to improve the accuracy of prediction of mineralization prospects and expanding the application of semantic mobile computing. We use five different semantic relations to build a semantic knowledge library, realize semantic retrieval, complete information extraction of geological text data, and study mineral profiles. Through the distributed database of mobile computing, the association rules and random forest algorithm are used to describe the characteristics of minerals and the ore-controlling elements, find the association rules, and finally combine the geological and mineral data of the area and use the random forest algorithm to realize the prospect of mineralization district forecast. The geological semantic model constructed in the article uses the knowledge library for associative search to achieve an accuracy rate of $87.9 \%$ and a recall rate of $96.5 \%$. The retrieval effect is much higher than that of traditional keyword retrieval methods. The maximum value of the posterior result of the mineralization prospect is 0.9027 , the average value is 0.0421 , and the standard deviation is 0.1069 . The picture is brighter, and the probability of mineralization is higher.

\section{Introduction}

Contemporary mineral resource evaluation is the modeling and evaluation process of complex high-dimensional nonlinear systems. Mineralization prediction is based on the guidance of scientific prediction theory, applying geological and mineralization theories and mathematical statistics to comprehensively study geology, geophysics, geochemistry, and remote sensing. We analyze mineralization geological conditions, summarize mineralization rules, establish comprehensive information mineralization prediction model, delineate and evaluate mineralization prospects, provide scientific basis for regional prospecting work deployment, and overall plan mineral resources development.

With the rapid development of computer technology, the digitization of geological information has become more and more popular, prompting the application of mathematical methods, mobile computing, and machine learning in mineralization prediction more and more. The prediction of mineralization prospects is the use of modeling and mobile calculation methods to continuously excavate information, such as geophysical and geochemical prospecting, focusing on the research of the information extraction process, and can truly understand the singularity of the enrichment and loss of elements in the mineralization process law, advanced and precise. We applying the learning method of the geological semantic model deterioration mobile computing machine to the prediction of mineral resources, aim to analyze how to efficiently use geological and mineral information under complex geological conditions, and realize the automation, intelligence, and accuracy of the prediction process of mineralization prospects. 
Lombardo et al. introduced a novel, semantic-based geological mapping method, and its application fields are the production of comprehensive geological maps of large administrative areas. Many methods for expressing geological knowledge through UML patterns and ontology have existed for more than ten years. These methods generate resources related to specific fields, such as lithology. They developed a conceptual model aimed at establishing digital codes for several areas of geological knowledge to support source interoperability. They used the term library designed to classify the elements of the geological map of the Alps in western Italy and the Apennines (Piedmont region) in the north. The digitally coded knowledge base is a group of merged ontology, called ontology. The encoding process identifies the semantically encoded objects, that is, geological units, and collects relevant information about these objects from authoritative resources (such as GeoSciML) (preferred to the application of SCHE). However, their conceptual model design is not applicable to applications in other regions [1]. Lamperti et al. effectively calibrate the agentbased model (ABM) to real data which is an open challenge. They combined machine learning and intelligent iterative sampling to clearly solve the problem of parameter space exploration and calibration of ABMs. This method "learns" a fast surrogate metamodel through a limited number of $\mathrm{ABM}$ evaluations and approximates the nonlinear relationship between ABM input (initial conditions and parameters) and output. The performance is evaluated according to the asset pricing model of Brock and Hommes (1998) and the "island" endogenous growth model (Fagiolo and Dosi, 2003). The results show that the machine learning agent obtained using the proposed iterative learning process provides a fairly accurate real model agent and greatly reduces the calculation time required for large-scale parameter space exploration and calibration. However, they have no specific settings in parameter design [2]. Lalomov et al.'s research established the heterogeneity of placer gold, reflecting the multistage history of the development of placer gold clusters and the diversity of bedrock mineralization formed by placer gold deposits. In the placer structure, three epochs are divided, reflecting the post-Palaeozoic development history of the Ural fold belt: (1) Mesozoic-Early Cenozoic quasiplain action, regional weathering crust formation, disintegration of primary gold sources, linear erosion-tectonics. The depression was formed, which accumulated material from the displaced weathering crust. (2) Primary mineral sources and intermediate gold deposits in the Pliocene-Quaternary orogenic tectonic activation and erosion stage. (3) Modern topography, water network, and alluvial deposits, the formation of placer. In terms of morphology, chemical composition, and internal structure of grains, five types of primary placer gold have been identified, three of which reflect the structure of the primary mineral source and have been almost completely eroded; the fourth records the quasiplanation stage and erosion traces of superficial changes in the development area of structural depressions. The fifth type has the typomorphic characteristics of near provenance gold, which is characterized by the mineralization exposed in the final stage of the morphological and structural develop- ment of the tectonic-magmatic Ural belt. Reconstructing the development stage of the Wagland Placer Group, it is possible to assess the potential of the existing placer and determine the prospect of economic primary mineralization. However, his research found diversity but did not fundamentally show the characteristics of its heterogeneity [3].

The innovations of this article are as follows: (1) fully construct a brand-new geological semantic model and complete the search of mining areas in combination with geological information; (2) use a mobile computing machine learning algorithm, mainly using a rule algorithm and a random forest algorithm, which is very good used in model construction; and (3) use five different semantic relations to construct a semantic knowledge base, realize semantic retrieval, complete information extraction of geological text data, and study mineral profiles.

\section{Prediction Method of Mineralization Prospect Based on Geological Semantic Model and Mobile Computer Machine Learning}

2.1. Mobile Computing. Mobile computing is a new technology that has emerged with the development of the Internet, mobile communications, distributed computing, databases, and other technologies $[4,5]$. Through mobile computer technology, computers and other intelligent terminal information devices can transmit data wirelessly, share resources, and provide customers with useful, accurate, and timely information at any time [6]. As a result, people's life and working methods have undergone great changes. Mobile information technology is an interacademic and extensive new technology. This is currently a hot field of computer technology research. In addition to network infrastructure, e-commerce, and software technical indicators, mobile computing is considered one of the important technologies that will have a broad impact on the future $[7,8]$. As a hotspot and emerging computing model, it is of great significance to study the problems, but there are also great challenges.

The computer environment is different from the previous distributed computer environment. In the traditional distributed computing environment, the location of the central computer is stable, the address information of the central computer is known, and each node maintains an uninterrupted connection through a fixed network, assuming that the network communication is symmetrical. In mobile computer systems, these assumptions no longer apply $[9,10]$. The entire environment of mobile computers is a distributed computer system composed of fixed nodes and mobile nodes. In this system, the user's location is not restricted by fixed equipment. The terminal can move freely and maintain the connection with each node of mobile communication through the network. This computing mode, which is not restricted by fixed networks and restricted by fixed hosts, provides great convenience for people to access information $[11,12]$.

Traditional distributed computer research is based on wired networks and fixed central computers. The search of 
the distributed database must be carried out under the conditions of fixed network connection, peer-to-peer communication cost, and fixed central computer node $[2,13]$. However, with the development of mobile computers and the popularity of portable devices, many computer nodes may create network connections in free loops, and the above assumptions are no longer valid $[14,15]$. Therefore, the concept of mobile computers and mobile databases was born, which became the new research direction of the international database community. Due to the characteristics and characteristics of the mobile computer environment, the distributed database and C/S (client/server) database in the database field cannot effectively support the mobile computer environment [16, 17]. To fully support portability, the computer needs to be improved or redesigned and upgraded to a new mobile database.

2.2. Semantic Technology. At present, the main semantic modeling technologies include semantic modeling technology based on semantic research and ontology-based semantic modeling technology. This article adopts a semantic modeling method based on logical relationships. The two technologies are introduced separately $[18,19]$.

The basic idea of the semantic web was first proposed by Tim Berners-Lee in 1998 who then formally proposed the concept of the semantic web at the XML2000 conference in 2000. The status of the semantic web was formally established when the W3C established the "Semantic Web Activity" organization in 2001 [20, 21]. The goal of the semantic network is to convert the information on the web page into the meaning of words that can be read by the computer system, so that smart devices can independently retrieve and access the information on the network effectively, thereby completing the application and higherlevel internet semantics knowledge application. Through the operation and processing of the semantic grammar form, the standardization of the semantic content is determined, and the operability at the semantic level is realized. In simple terms, semantics is used to implement a logical architecture [22, 23].

Ontology-based semantic modeling methods are also based on the semantic web, to better solve the sharing and reuse of information on the semantic level. Ontology or ontology is originally a philosophical concept, used to study the essence of the objective world and is a systematic explanation and explanation of objective existence. The application of semantic modeling methods in the field of artificial intelligence can clearly indicate the standardization and specification of all shared conceptual models. Ontologybased modeling includes the following five basic modeling primitives: class, relationship, function, axiom, and instance.

The logical relationship-based method defines a nested data model for expressing data relationships and a set of SQL-like description languages for describing the logical relationship between data. In the process of semantic analysis, the Chase method is used to continuously compare the data. We track the existing constraint relationships until the largest semantic set is generated, so as to obtain complete semantic information.
2.3. Machine Learning. Random forest $(\mathrm{RF})$ is a general data extraction method in the field of machine learning. This is a typical multiclass algorithm. Decision tree is the main classification method of random forest algorithm. The essence of a decision tree is actually a tree composed of many nodes. The basic principle of the random forest algorithm is to use technical sampling to form a new random sampling training group and then use an autonomous data set to model the decision tree, form a random forest, and vote for the classification result.

The random forest algorithm combines the initial sampling method based on the decision tree algorithm theory, collects multiple independent tree classifiers, and sorts and predicts the final result through a voting strategy. The random forest algorithm has the characteristics of theoretically easy to understand, low adaptability parameters, and strong antinoise function. The most important thing is that it has high classification efficiency in practical applications and is not easy to cause overload. Because there is no need to understand the excellent random performance and the performance of historical samples, it is widely used in many fields. Therefore, many researchers have conducted extensive research and applications on random forests.

The random forest algorithm has excellent antinoise ability and outlier ability. The random forest algorithm does not need to preprocess the classification object, so it simplifies the preprocessing procedure of related data. The random forest algorithm has excellent performance in many aspects, but it still has shortcomings. For example, when selecting features, the random forest algorithm will randomly select from the data set, and the parameter selection of the random forest algorithm is manually set. These functions do not show the error that increases the classification result. From the perspective of practical applications, it is necessary to further enhance the ability of the random forest algorithm to output high-quality features, optimize parameter selection, further reduce the generalization error of the random forest model, and improve the accuracy of the random forest algorithm classification [24].

\section{Prediction Model of Mineralization Prospect Based on Geological Semantic Model and Mobile Computer Machine Learning}

\subsection{Geological Semantic Model}

3.1.1. Construction of Semantic Model of Geological Data. This paper mainly constructs a geological semantic model based on the meaning of the semantic model and proposes a four-element combination, which are a collection of geological data concepts, a collection of geological relations, a collection of geological attributes, and a collection of examples, which are represented by GDO, GDR, GDP, and GDI [25]. According to the research status of geological ontology, according to the types of geological work and the application of geological data, in order to realize the functions of compilation, analysis, reflection, restoration, and intelligent advancement of geological data, various classification systems have been classified into many subcategories. 
3.1.2. Semantic Relationship of Geological Semantic Model Application. The geological semantic model constructed in this paper is mainly applied to five kinds of semantic relations, namely the following: (1) subordinate relation: mainly the concept level problem in the model and the subordination relation; (2) equivalence relation: the concept of the same level or the equivalence relation; (3) cross-relationship: that is, there are some identical conceptual relationships between two different geological concepts; (4) concept instance relationship: used to more clearly illustrate the relationship between various concepts and corresponding cases; and (5) spatial relationship: mainly refers to the inclusion, the facts of the positional relationship of the phase relationship, such as cutting off, connecting, and integrating.

3.1.3. Semantic Retrieval. First of all, we need to collect geological data and build a basic dictionary of geology and mineral resources in order to achieve semantic retrieval. There are two main sources of data: one is the professional knowledge in the geological field; the other is the mining of geological data through mobile computer learning technology [26].

Here, the user's needs are an entity. Through data preprocessing, the user only needs to enter understandable keywords and information to obtain intelligent, accurate, and user-friendly data results, which have undergone data fusion and data preprocessing. And other related processes achieve the semantic conversion of data information, to meet the needs of users.

3.1.4. Functions Realized by Geological Semantic Model. Functions realized by geological semantic model are as follows: (1) Coarse-grained geological information knowledge serves geological text clustering. When the user retrieves the relevant geological information, the relevant conditions and grouping results will be automatically saved, which is conducive to the user's second search; (2) comprehensive semantic retrieval of geological data and geological literature information. Semantic retrieval includes text and tables, which requires solving these two data fusion problems in the process of establishing a database; (3) display and obtain content information based on complete text; (4) the comprehensive problem of spatial data and nonspatial data. It can solve the nonspatial data problem of the system and complete the visual representation of spatial data; (5) association analysis: research on geology not only is limited to geological knowledge but also includes related information such as authors, units, projects, and mineral resources and conducts correlation analysis.

\subsection{Prediction of Mineralization Prospects Based on Geological Semantic Model and Mobile Computer Machine Learning}

3.2.1. Right of Evidence Law. The weight of evidence method is to use a certain ore-controlling mineralization geological condition or prospecting information sign as a predictive factor in the mineralization prediction. The predictive factor is the evidence factor, and the weight value is used to quantitatively measure the importance of each evidence factor to the mineralization. Then, each evidence factor is assigned a binary value according to the two states of the evidence factor "existence" and "nonexistence." The most important and critical step in the calculation of mineralization prediction using the weight of evidence method is to determine the weight of evidence for these evidence factors and the importance of mineralization. The model of the right of evidence is

$$
\operatorname{EVI}\left(F \mid B_{1} B_{2} \cdots B_{n}\right)=Q_{O}+Q_{1}+\cdots+Q_{n}
$$

In the formula, $F$ is the event to be predicted, $B_{1} B_{2} \cdots B_{n}$ is the predictor, $Q$ is the prior probability of the predicted event $F$, and $Q_{1}, Q_{2}, \cdots Q_{n}$ is the corresponding weight value. The weight value is divided into positive full $Q+$ and negative right $Q$ - two kinds.

According to the existence or nonexistence of each evidence factor, the corresponding weight value is calculated. $Q+$ represents the weight value of the existing state, and $Q$ - represents the weight value of the nonexistent state. The calculation of mineralization prediction based on the weight of evidence method is roughly divided into three steps: first, calculate the probability of occurrence of deposits (points) in the study area according to the principle of probability theory; secondly, calculate the corresponding weight values according to the two states of each factor; and finally, all the evidence factors involved in the prediction are integrated to calculate the posterior probability value.

To apply the weight of evidence method, the research area must be divided into cells first. The number of deposit points in the research area is set as $F$. From the principle of probability theory, it can be obtained that any cell in the research area can be selected. This cell contains the deposit points the probability: $L(F)=F / N . L(F)$ is the prior probability of the study area, into the form of chance.

$$
I(F)=\frac{L(F)}{[1-L(F)]} .
$$

The probability of mineral occurrence in the presence of evidence factor B is obtained as

$$
L(F \mid B)=L(B \cap F) / L(B)=N(B \cap F) / F .
$$

From Equation (3), it can be obtained that the posterior probability of the occurrence of a mining point in the presence of the evidence factor $B$ is

$$
L(F \mid B)=\frac{L(B \mid F)}{L(B)} \times L(F)
$$

From Equation (4), it can be concluded that the posterior probability can actually be understood as the conditional probability of the existence of the mine in the presence of the evidence factor $B$. In the same way, the posterior probability when factor $B$ does not exist is

$$
L(F \mid \bar{B})=\frac{L(\bar{B} \mid F)}{L(\bar{B})} \times L(F) .
$$


3.2.2. Definition and Calculation of Weight. The importance of each evidence factor to mineralization is quantitatively analyzed by the weight value of the evidence factor. If the weight of evidence method is used to carry out mineralization prediction, then it is necessary to calculate the weight value of each evidence factor to the mineralization, and the formula is as follows:

$$
\begin{gathered}
Q^{+}=\ln \frac{L(B \mid F)}{L(B \mid \bar{F})}, \\
Q^{-}=\ln \frac{L(B \mid F)}{L(B \mid \bar{F})} .
\end{gathered}
$$

In the above formula, $Q+$ and $Q-$, respectively, represent the weight values of the evidence factor in the "existence" and "nonexistence" states in the weight of evidence model. In (6), $Q+$ represents the weight value in the state of existence, and in (7), $Q$ - represents the size of the weight value in the absence of state.

3.2.3. Comprehensive Multiple Evidence Factors. In the actual mineralization prediction, due to the complexity of the mineralization and the accuracy of the prediction results, we need to use multiple predictive factors. At this time, we need to comprehensively calculate multiple evidence factors and calculate the posterior probability of multiple evidence factors. The method and steps are the same as when calculating one evidence factor in the previous section. For example, in a certain mineralization prediction, there are $n$ factors related to mineralization, which are represented as $1,2, \ldots, n$. According to the calculation steps in the previous section, the posterior probability of occurrence or nonoccurrence of deposit point $F$ under the existence of these $n$ evidence factors can be obtained as

$$
\begin{gathered}
L\left(F \mid B_{1} B_{2} \cdots B_{n}\right)=\frac{L\left(B_{1} B_{2} \cdots B_{n} \mid F\right)}{L\left(B_{1} B_{2} \cdots B_{n}\right)} L(F), \\
L\left(\bar{F} \mid B_{1} B_{2} \cdots B_{n}\right)=\frac{L\left(B_{1} B_{2} \cdots B_{n} \mid \bar{F}\right)}{L\left(B_{1} B_{2} \cdots B_{n}\right)} L(\bar{F}) .
\end{gathered}
$$

The posterior probability is converted to the probability form to get

$$
I\left(F \mid B_{1} B_{2} \cdots B_{n}\right)=\frac{L\left(B_{1} B_{2} \cdots B_{n} \mid F\right)}{L\left(B_{1} B_{2} \cdots B_{n} \mid \bar{F}\right)} I(F) .
$$

Assuming that the evidence factors are conditionally independent, the probability of the occurrence of the evidence factors under the condition of the existence of the mine $F$ can be obtained as

$$
L\left(B_{1}, B_{2}, \cdots, B_{n} \mid F\right)=L\left(B_{1} \mid F\right) L\left(B_{2} \mid F\right) \cdots L\left(B_{n} \mid F\right) .
$$

In the same way, it can be obtained that when the mine point $F$ does not exist, the probability of occurrence of the evidence factor is

$$
L\left(B_{1}, B_{2}, \cdots, B_{n} \mid \bar{F}\right)=L\left(B_{1} \mid \bar{F}\right) L\left(B_{2} \mid \bar{F}\right) \cdots L\left(B_{n} \mid \bar{F}\right) .
$$

From the above two formulas, the probability formula can be obtained, which can be expressed as

$$
I\left(F \mid B_{1} B_{2} \cdots B_{n}\right)=\frac{L\left(B_{1} \mid F\right) L\left(B_{2} \mid F\right) \cdots L\left(B_{n} \mid F\right)}{L\left(B_{1} \mid \bar{F}\right) L\left(B_{2} \mid \bar{F}\right) \cdots L\left(B_{n} \mid \bar{F}\right)} \times I(F) .
$$

The logarithmic transformation of the above formula obtains the posterior probability of the occurrence of the deposit point $F$ under the condition of the existence of n evidence factors as

$$
\operatorname{EVI}\left(F \mid B_{1} B_{2} \cdots B_{n}\right)=\operatorname{EVI}(F)+Q_{1}^{+}+Q_{2}^{+}+\cdots Q_{n}^{+} .
$$

According to the mathematical symbol of sum, the above formula can be simplified to

$$
\begin{gathered}
\operatorname{EVI}\left(F \mid B_{1} B_{2} \cdots B_{n}\right)=\operatorname{EVI}(F)+\sum_{j=1}^{n} Q_{j}^{k}, \\
Q_{j}^{k}=\left\{\begin{array}{l}
Q^{+} \\
Q^{-} .
\end{array}\right.
\end{gathered}
$$

The weight when the evidence factor exists is $Q+$, and the weight when the evidence factor does not exist is $Q-$. The formula as a posterior probability is expressed as follows:

$$
L\left(F \mid B_{1}^{k} \cap B_{2}^{k} \cdots B_{n}^{k}\right)=\frac{\exp \left\lfloor\operatorname{EVI}\left(F \mid B_{1}^{k} \cap B_{2}^{k} \cdots B_{n}^{k}\right)\right\rfloor}{1+\exp \left[\operatorname{EVI}\left(F \mid B_{1}^{k} \cap B_{2}^{k} \cdots B_{n}^{k}\right)\right]} .
$$

Fuzzy weight calculation formula is as follows.

$$
S_{\lambda B(x)}=\ln \frac{L\left[\lambda_{B(x)} \mid F\right]}{L\left[\lambda_{B(x)} \mid \bar{F}\right]}=\ln \frac{\lambda_{B(x)} L\left(B_{1} \mid F\right)+\left[1-\lambda_{B(x)}\right] L\left(B_{2} \mid F\right)}{\lambda_{B(x)} L\left(B_{1} \mid \bar{F}\right)+\left[1-\lambda_{B(x)}\right] L\left(B_{2} \mid \bar{F}\right)} .
$$

Among them, the membership function can be calculated from the following linear relationship.

$$
\lambda_{B}(x)=\frac{V-\min _{x \in B_{2}} V}{\max _{x \in B_{2}} V-\min _{x \in B_{2}} V} .
$$

Fuzzy weights can explain the importance of various forms of uncertain factors, while ordinary weight of evidence can only explain the importance of the existence or nonexistence of uncertain factors, so fuzzy weights can be more comprehensive explain the importance of the factor. According to the calculation steps of the ordinary weight of evidence method, we can get the posterior probability of 
the fuzzy weight of evidence method as

$$
\operatorname{EVI}\left(F \mid B_{1}^{k} \cap B_{2}^{k} \cdots B_{n}^{k}\right)=\operatorname{EVI}(F)+\sum_{j=1}^{n} Q_{j}^{k}
$$

In the above formula, $B_{1}^{k}, B_{2}^{k}, \cdots B_{n}^{k}$ represent the evidence factors of $n$ different states and $Q_{j}^{k}$ the corresponding weight values of these factors in different states. Since the calculation of the weight value of the fuzzy weight of evidence first needs to calculate the two extreme sets and remove these two extreme combinations, then it should correspond to a smaller subset, so formula (20) only needs to satisfy the condition of independence in a smaller range. Therefore, the conditional independence required to apply the fuzzy weight of evidence method is weaker than that of the ordinary weight of evidence. This also shows that the fuzzy weight of evidence method reduces the overall error of the posterior probability. Then it can be said that compared with ordinary evidence rights methods, to a certain extent, fuzzy evidence rights avoid conditional independence.

\section{Prediction and Analysis of Mineralization Prospects Based on Geological Semantic Model and Mobile Computer Learning}

4.1. Comparison of Experimental Results of Geological Semantic Model. In the process of semantic retrieval, the geological semantic model established in this paper can not only use keywords but also use related search and thesaurus retrieval, which improves the accuracy and comprehensiveness of the search. Hierarchy excavates the rules and knowledge of keywords, supplements, and expands related knowledge searches. It can be seen from Table 1 that using the thesaurus and knowledge map to search is more accurate and comprehensive than keyword retrieval. The knowledge map finds a large number of materials, and the accuracy rate reaches $87.9 \%$. The rate reached $96.5 \%$.

We have adopted three fusion methods: PCA fusion, IHS fusion, and wavelet fusion, respectively, fusion processing of $\mathrm{R}, \mathrm{G}$, and $\mathrm{B}$ multispectral data, to maximize the role of the data. From Figure 1, we can see that the wavelet transform method is superior to other fusion methods in terms of spectral information retention and peak signal-to-noise ratio for the statistical calculation of the amount of information after fusion, so this time, the wavelet transform method is used for fusion processing.

4.2. Spectral Characteristics of Rock and Mineral Alteration. Different information such as minerals, rocks, or alteration and mineralization have different characteristic absorption spectrum and characteristic reflection spectrum characteristics, so that different geological bodies or geological phenomena are directly reflected as different spectrum curves on remote sensing images. With the improvement of spectral resolution, the spectral characteristics of rock minerals have become a favorable tool for remote sensing mineralization information extraction, and they play an increasingly important role in the process of detailed exploration and mineralization prediction.

The ions and groups corresponding to the spectral characteristics of various rock minerals are shown in Table 2. The spectral characteristics within the range of $1.3 \sim 2.5 \mu \mathrm{m}$ are mainly affected by carbonate ions, hydroxide ions, and possible water molecules in the minerals. The absorption band of $\mathrm{CO}^{32}$ - is the strongest at $2.35 \mu \mathrm{m}$, corresponding to the 8th band of ASTER, which can be used to detect the presence of carbonate minerals. The absorption band of $\mathrm{OH}$ - is stronger at $1.4 \mu \mathrm{m}$ and $2.2 \mu \mathrm{m}$. Since $1.4 \mu \mathrm{m}$ is not within the spectral range of the ASTER data, the absorption characteristics of the 6th band corresponding to $\mathrm{OH}-$ at $2.2 \mu \mathrm{m}$ and $2.3 \mu \mathrm{m}$ are often used in the ASTER data to identify minerals.

The altered minerals formed by cyanide rock formation include chlorite, carbonated minerals, albite, and epidote. The related minerals include $\mathrm{Au}, \mathrm{Cu}, \mathrm{Pb}, \mathrm{Zn}, \mathrm{Fe}$, and other metal minerals. According to the analysis results of the mineral spectrum characteristics, the 6 bands (Band1, Band2, Band3, Band5, Band8, and Band9) of the ASTER data are combined to extract the green rock formation alteration information [27]. The negative high value of the 8th band and the positive high value of the 9th band are used as the criterion of the alteration information eigenvector. It can be seen from the eigenvector matrix of Figure 2 that the main contribution of the fifth principal component (PC5) comes from Band8 (-0.63562) and Band9 (0.66101), satisfying the judgment criterion. Therefore, the highlight area of the PC5 image is the area with strong chlorite and carbonate alteration.

It can be seen from Figure 3 that each element has a higher extreme value, and the second and third highest values have a large change gradient, and the standard deviation and coefficient of variation are also large. Based on the statistics of the characteristic value of each element in the study area, the content distribution histogram and the content logarithmic distribution histogram of each element are, respectively, analyzed. The results show that due to the existence of extremely high and extremely low values, whether each element is difficult for the original value or the logarithmic value to obey the normal distribution, the iterative processing method is used to deal with the extreme outliers of the element data.

4.3. Statistical Analysis of the Weight Coefficient of the Evidence Layer. We set 7 evidence layer numbers, all of which belong to the element anomaly distribution map. The letters indicate the meaning: (a) Ag element, (b) Au element, (c) Cr element, (d) Cu element, (e) Mo element, (f) Ni, and (g) $\mathrm{Pb}$.

It can be seen from Figure 4 that all the layers we set meet the conditional test. We, respectively, estimated the positive and negative weight coefficients, variances, partial variances, etc. of these evidence layers. The estimation results are shown in Figure 4.

We conducted regression analysis on the abnormal distribution of nine types of elements with different iteration times. In the process of parameter optimization using 
TABLE 1: Comparative verification and analysis of semantic retrieval experiments.

\begin{tabular}{lccc}
\hline Search method & Number of obtained materials & Find accuracy, \% & Find comprehensive rate, \% \\
\hline Keyword search & 265 & 78.5 & 46.7 \\
Semantic retrieval (thesauri) & 412 & 90.6 & 82.1 \\
Semantic retrieval (knowledge illustrated book) & 456 & 87.9 & 96.5 \\
\hline
\end{tabular}

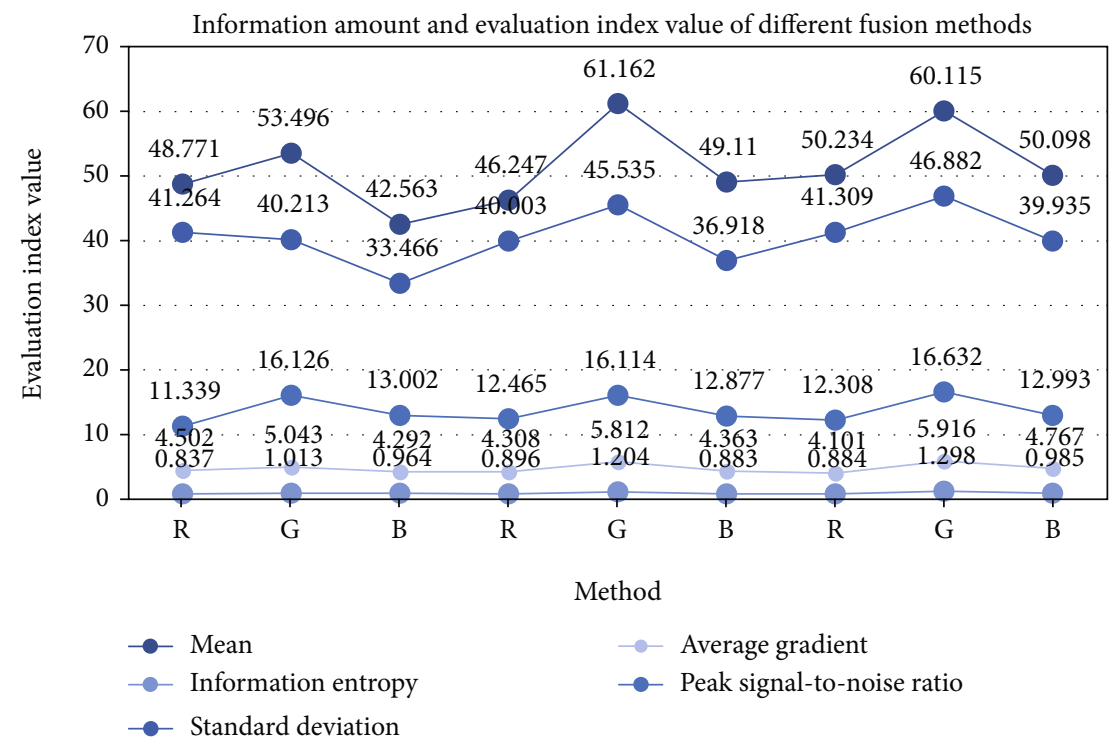

Figure 1: Information amount and evaluation index value of different fusion methods.

TABLE 2: Ion and group absorption bands corresponding to the characteristics of rock mineral reflection spectrum.

\begin{tabular}{|c|c|c|c|}
\hline Ion or group & $\begin{array}{c}\text { Characteristic absorption band } \\
\text { center }\end{array}$ & $\begin{array}{l}\text { Corresponding ASTER } \\
\text { band }\end{array}$ & Typical minerals \\
\hline Iron ion $\mathrm{Fe}^{2+}$ & $0.45,0.52,0.55,1.0 \sim 1.1$ & Band1 (0.52 0.60) & Lepidocrocite \\
\hline Iron ion $\mathrm{Fe}^{3+}$ & $0.49,0.70,0.87$ & $\begin{array}{l}\text { Band2 }(0.61 \sim 0.69) \\
\text { Band3 }(0.76 \sim 0.86)\end{array}$ & Goethite, hematite, jarosite \\
\hline $\begin{array}{l}\text { Hydroxyl(OH-)Al-OH, Mg- } \\
\mathrm{OH}\end{array}$ & $1.4,2.2,2.3$ & $\begin{array}{l}\text { Band6 }(2.185 \sim 2.225) \\
\text { Band7 }(2.225 \sim 2.285)\end{array}$ & $\begin{array}{c}\text { Kaolinite, pyrophyllite, muscovite, talc, } \\
\text { serpentine }\end{array}$ \\
\hline & & Band6 (2.185 2.225) & \\
\hline Carbonate ion $\mathrm{CO}^{3 \sim}$ & $1.9,2.0,2.16,2.35,2.55$ & $\begin{array}{l}\text { Band7 }(2.225 \sim 2.285) \\
\text { Band8 }(2.295 \sim 2.285)\end{array}$ & Calcite, dolomite \\
\hline
\end{tabular}

conjugate gradient, we tested the predictions of $30,50,80$, and 100 search iterations, respectively. The effect, the calculation result of the regression coefficient, is shown in Figure 5. It can be seen from Figure 5 that when the parameter optimization is iterated to 80 and 100 times, the regression coefficient is very close and basically reached a stable state.

In order to compare the evidence-weighted model, the logistic regression model, and the constrained Boltzmann machine model for the mineralization prediction effect, the Youden index is also defined as the sum of the TP rate and the FP rate minus 1, the threshold increment interval is defined, and three types are calculated, respectively. The model predicts the Youden index of the metallogenic unit, and the threshold corresponding to the maximum Youden index is used as the threshold for the corresponding model to determine the metallogenic unit. After obtaining the corresponding judgment threshold, the AUC value and the standard deviation SEAUC value of the three models were calculated using formulas (3) and (5), and the significance level $\alpha=0.05$ was set to calculate the corresponding ZAUC value. The results are shown in Figure 6.

4.4. Experimental Prediction Results of Mineralization Prospects. Figure 7 shows the typical linear and circular fault trace characteristics in the study area and nearby areas. The intrusive body in Figure 7(a) is the Cenozoic Eocene strata; the left side in Figure 7(b) is the Mesozoic Triassic intrusion 


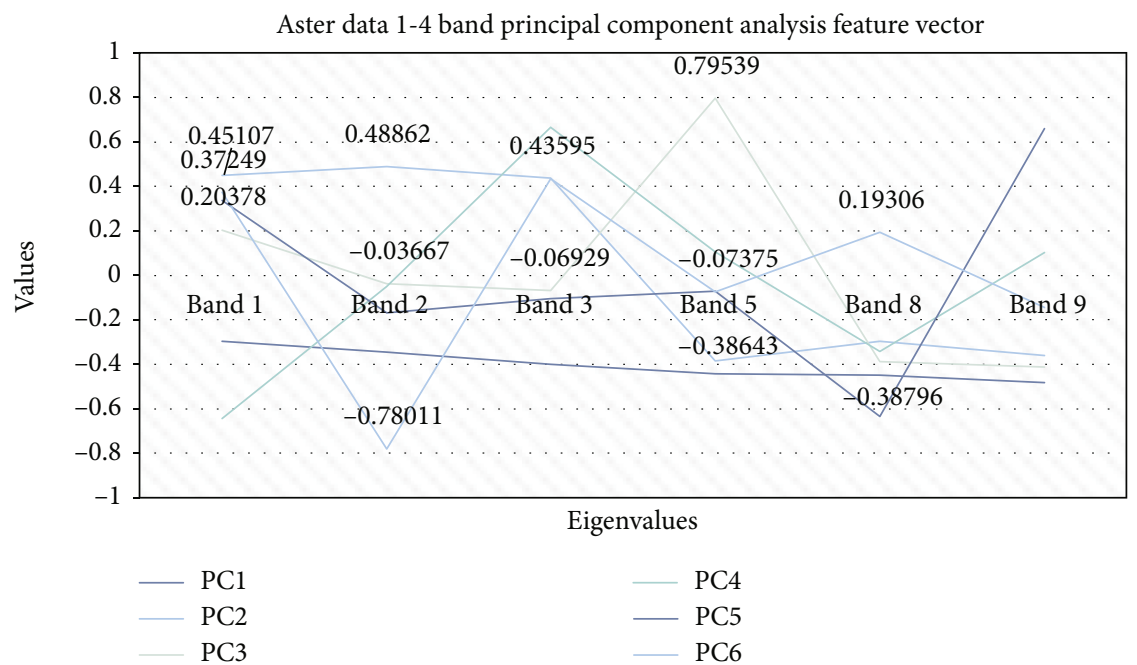

FIGURE 2: ASTER data 1-4 band principal component analysis feature vector.

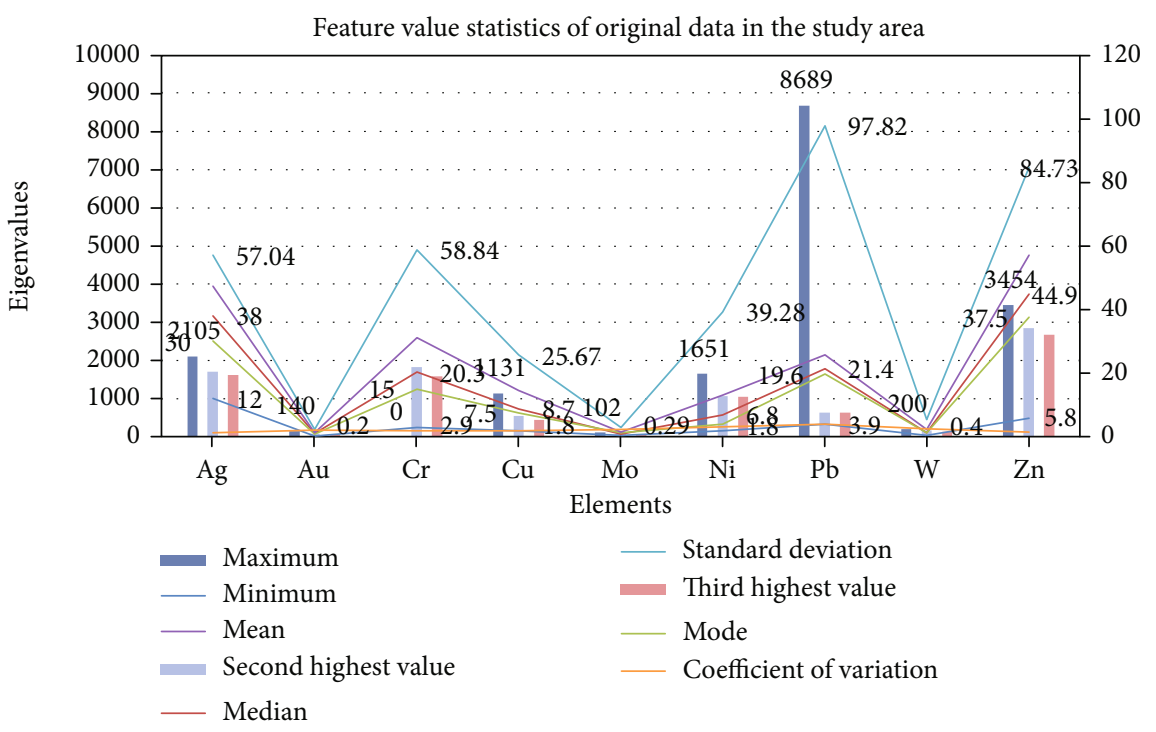

FIGURE 3: Feature value statistics of original data in the study area.

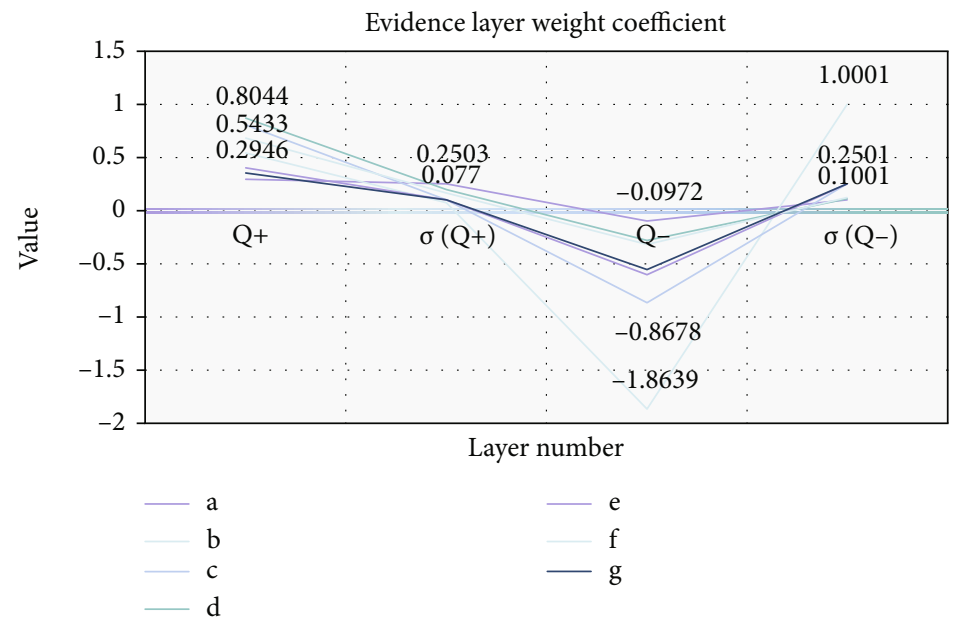

FIGURE 4: Evidence layer weight coefficient. 


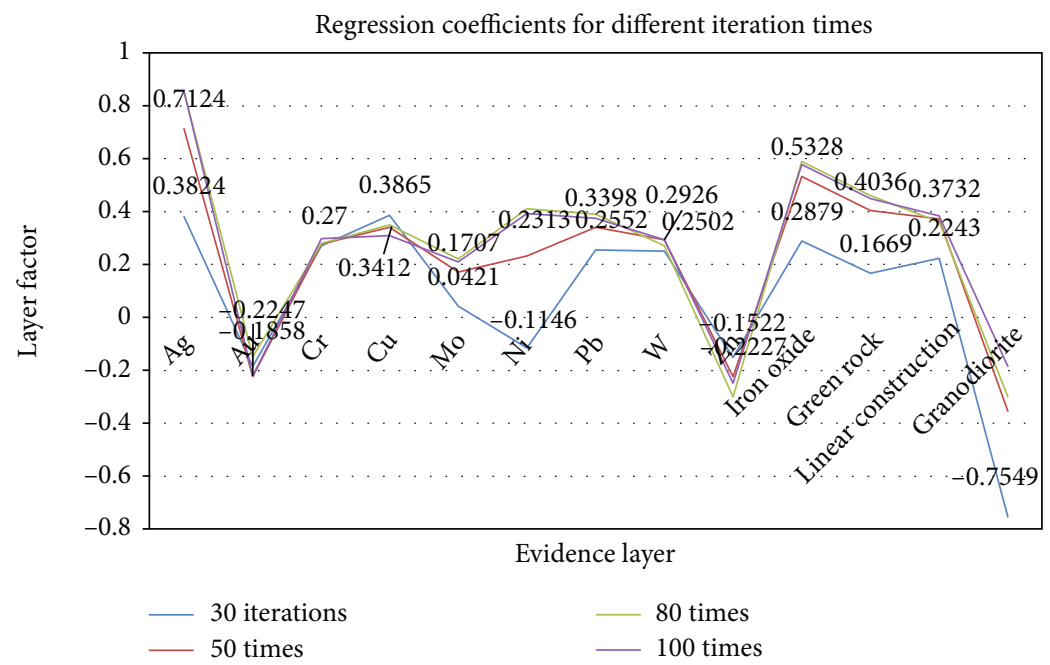

FIgURE 5: Regression coefficients for different iteration times.

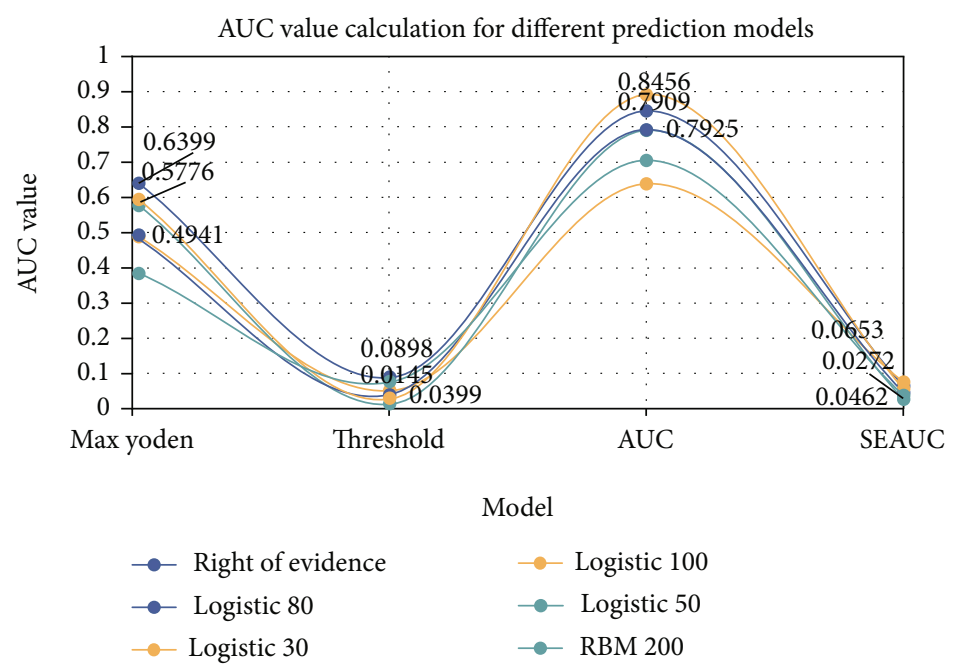

Figure 6: AUC value calculation for different prediction models.

body, and the right side is the fold formed during the Jurassic-Cretaceous period; Figure $7(\mathrm{c})$ is the fault divided into many triangular fault cliffs form fault triangles; Figure $7(\mathrm{~d})$ shows the fractured fracture zone accompanying the formation of large faults, mostly small parallel fractures; Figure 7(e) shows the interruption and loss of the Upper Cretaceous (Ks2c) strata; Figures 7(e) and 7(f) are the displacement of the Jurassic stratum, and there are faults.

From the slope of the broken line in Figure 8, it can be seen that the ring structure within the range of $0-0.5 \mathrm{~km}$ contains $50 \%$ of the mine points, and the formation of minerals in this area is mainly affected by the ring structure; the secondary faults within the range of $1-1.5 \mathrm{~km}$ include the number of mine points has risen sharply, including nearly $43 \%$ of the mine points, and the largescale faults have always been at a value of 0 , indicating that the secondary faults in this range play a major role in controlling the formation of the deposit; within the range of $1.5-3 \mathrm{~km}$, the secondary faults have a major control effect on the formation of the deposit. The rate of increase in the number of ore points contained in the fault exceeds the ring structure, indicating that the formation of ore bodies in this range is mainly controlled by secondary faults; and the influence of large faults on the formation of ore deposits is in the range of $4-6 \mathrm{~km}$ and $8-10 \mathrm{~km}$. In general, the relationship between the structure of the study area and the known ore sites reflects the characteristics of the structure controlling the location of the deposit.

Based on the optimized weight of evidence algorithm module, the seven weight of evidence factors are superimposed and analyzed, and the final result of the posterior probability of mineralization in the study area is shown in Figure 9. The minimum is 0 , the maximum is 0.902722 ; the mean is 0.042112 , and the standard deviation is 0.106953 . The picture is brighter, and the probability of mineralization is higher. 


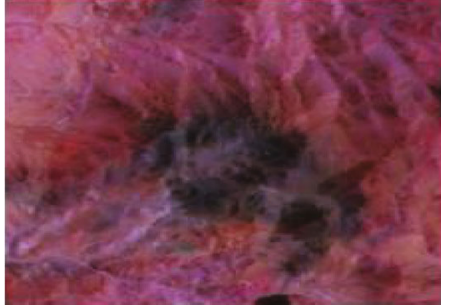

(a)

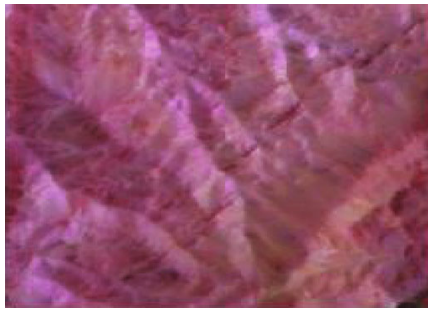

(d)

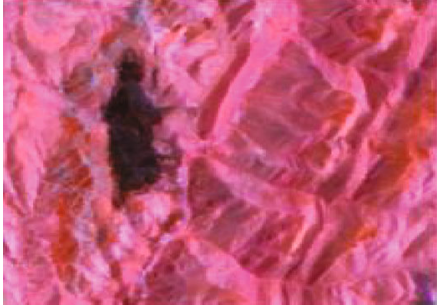

(b)

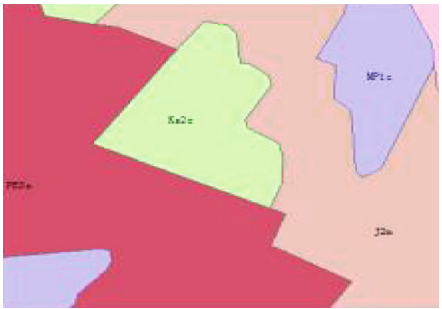

(e)

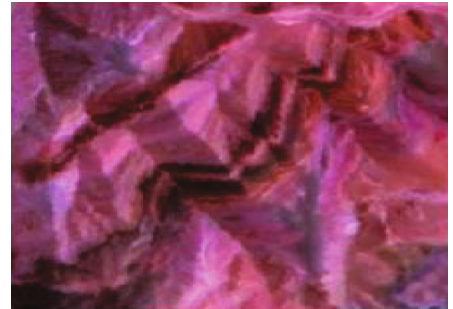

(c)

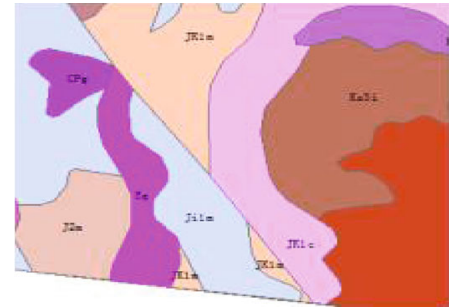

(f)

FIGURE 7: Characteristic map of linear and circular fault tracks (the picture from Baidu Gallery). (a) Semicircular intrusion body. (b) Intrusion + folds. (c) Fault triangle. (d) Broken shear zone. (e) Disruption and lack of formation. (f) Displacement of faults in the same stratum.

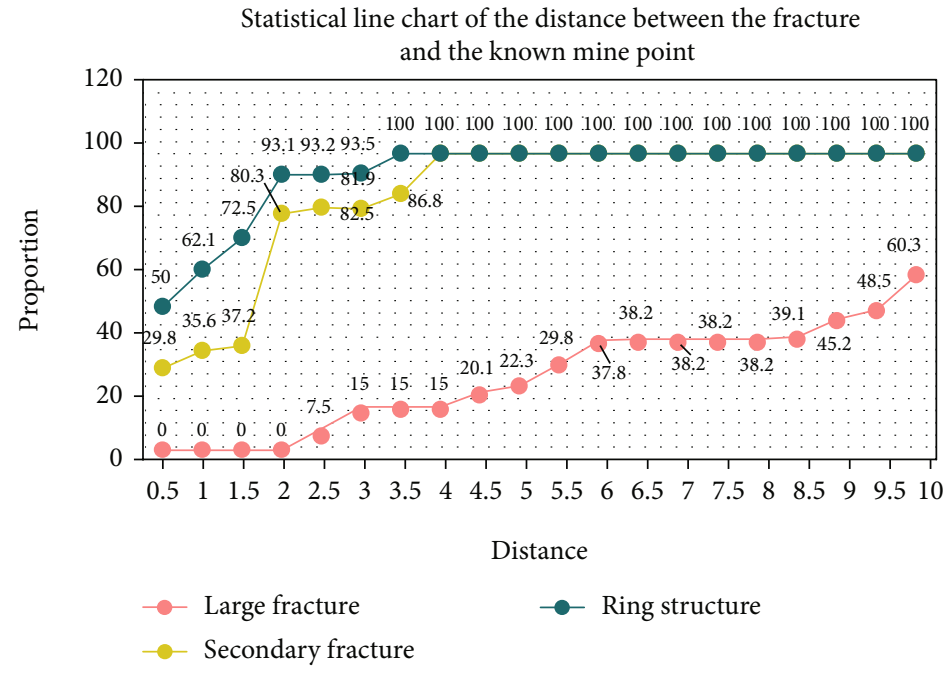

FIGURE 8: Statistical line chart of the distance between the fracture and the known mine point.

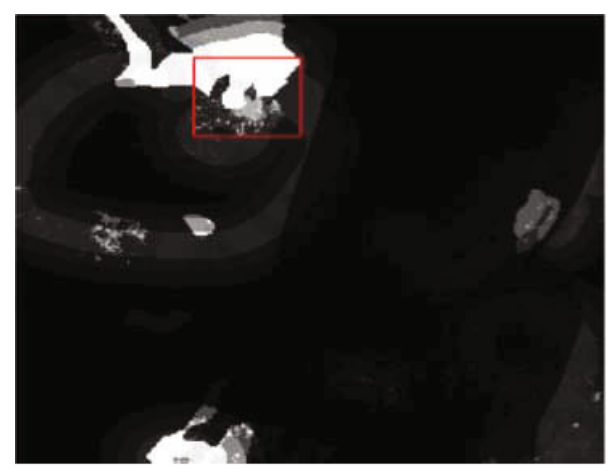

FIGURE 9: Result map of the posterior probability of mineralization in the study area (the picture from Baidu Gallery).

\section{Conclusions}

This article is mainly based on the study of geological semantic model and mobile computing machine learning to predict the mineralization prospects, constructs the geological semantic model, uses the rule algorithm and random forest algorithm in machine learning, and completes the collection and preprocessing of geological information which achieved a high recall rate and precision rate. We analyzed the geological conditions of mineralization, summarized the rules of mineralization, established a prediction model of mineralization prospects based on geological semantic model and mobile computer learning, and realized the prediction of prospects of mineralization, which is conducive to the deployment of prospecting work and the development 
and utilization of mineral resources overall planning. The innovation of this paper is to fully construct a brand-new geological semantic model, complete the search of the mining area in combination with geological information, and use the mobile computing machine learning algorithm, mainly using the rule algorithm and the random forest algorithm, which are well applied to the model construction. The shortcomings of this article are the limitations of working conditions and the influence of the number of studies. It is hoped that it can be more perfect in the future research work to improve the accuracy of the research results.

\section{Data Availability}

No data were used to support this study.

\section{Conflicts of Interest}

The authors declare that they have no conflicts of interest.

\section{Acknowledgments}

This work was sponsored in part by the Liaoning Provincial Department of Education's Young Scientific and Technological Talent "Nurturing" Project (LJ2020QNL003) and the 2020 Fuxin Social Science Research Project (2020Fsllx074).

\section{References}

[1] V. Lombardo, F. Piana, and D. Mimmo, "Semantics-informed geological maps: conceptual modeling and knowledge encoding," Computers \& Geosciences, vol. 116, pp. 12-22, 2018.

[2] F. Lamperti, A. Roventini, and A. Sani, "Agent-based model calibration using machine learning surrogates," Journal of Economic Dynamics \& Control, vol. 90, pp. 366-389, 2018.

[3] A. V. Lalomov, V. A. Naumov, A. V. Grigorieva, and L. O. Magazina, "Evolution of the Vagran gold-bearing placer cluster (northern Urals) and prospects for revealing bedrock mineralization," Geology of Ore Deposits, vol. 62, no. 5, pp. 407$418,2020$.

[4] Y. Lei, Z. Zhu, and Q. Li, “An ontological metamodeling framework for semantic simulation model engineering," Journal of Systems Engineering and Electronics, vol. 31, no. 3, pp. 527-538, 2020.

[5] L. Lalicic and A. Dickinger, "An assessment of user-driven innovativeness in a mobile computing travel platform," Technological Forecasting and Social Change, vol. 144, pp. 233$241,2019$.

[6] H. Gao and Y. Yin, "Editorial: ACM/Springer mobile networks \& applications-special issue on mobile computing and software engineering," Mobile Networks and Applications, vol. 25, no. 2, pp. 672-673, 2020.

[7] L. YIN, Y. GUO, H. ZHANG, W. HUANG, and B. FANG, "Threat-based declassification and endorsement for mobile computing [J]," Chinese Journal of Electronics, vol. 28, no. 5, pp. 1041-1052, 2019.

[8] S. K. Singh and D. P. Vidyarthi, "A heuristic channel allocation model with multi lending in mobile computing network," International Journal of Wireless and Mobile Computing, vol. 16, no. 4, pp. 322-339, 2019.
[9] C. Z. Lu, G. S. Zeng, and Y. J. Xie, "Bigraph specification of software architecture and evolution analysis in mobile computing environment," Future Generation Computer Systems, vol. 108, pp. 662-676, 2020.

[10] T. Yang, Z. Jiang, R. Sun, N. Cheng, and H. Feng, "Maritime search and rescue based on group mobile computing for unmanned aerial vehicles and unmanned surface vehicles," IEEE Transactions on Industrial Informatics, vol. 16, no. 12, pp. 7700-7708, 2020.

[11] B. Liao, Y. Ali, S. Nazir, L. He, and H. U. Khan, "Security analysis of IoT devices by using mobile computing: a systematic literature review," IEEE Access, vol. 8, pp. 120331-120350, 2020.

[12] C. Helma, T. Cramer, S. Kramer, and L. De Raedt, "Data mining and machine learning techniques for the identification of mutagenicity inducing substructures and structure activity relationships of noncongeneric compounds," Journal of Chemical Information and Computer Sciences, vol. 35, no. 4, pp. 1402-1411, 2018.

[13] N. Zimmerman, A. A. Presto, S. P. N. Kumar et al., "A machine learning calibration model using random forests to improve sensor performance for lower-cost air quality monitoring," Atmospheric Measurement Techniques, vol. 11, no. 1, pp. 291-313, 2018.

[14] I. Goodfellow, P. Mcdaniel, and N. Papernot, "Making machine learning robust against adversarial inputs," Communications of the ACM, vol. 61, no. 7, pp. 56-66, 2018.

[15] S. L. Benjamin, "Inverse molecular design using machine learning: generative models for matter engineering," Science, vol. 361, no. 6400, pp. 360-365, 2018.

[16] V. L. Kornienko, G. A. Kolyagin, G. V. Kornienko, and T. A. Kenova, "The prospects of the in situ and ex situ use of aqueous solutions of hydrogen peroxide electrogenerated from oxygen," Russian Journal of Electrochemistry, vol. 56, no. 5, pp. 405-417, 2020.

[17] R. Ghezelbash, A. Maghsoudi, M. Daviran, and H. Yilmaz, "Incorporation of principal component analysis, geostatistical interpolation approaches and frequency-space-based models for portraying the $\mathrm{Cu}$-Au geochemical prospects in the Feizabad district, NW Iran," Geochemistry, vol. 79, no. 2, pp. 323336, 2019.

[18] L. I. ZhenZhen, Key Laboratory of Mineral Resources, Institute of Geology and Geophysics, Chinese Academy of Sciences, Beijing 100029, China, Institutions of Earth Science, Chinese Academy of Sciences, Beijing 100029, China et al., "Basic characteristics, research progresses and prospects of Sn-Ag-base metal metallogenic system," Acta Petrologica Sinica, vol. 35, no. 7, pp. 1979-1998, 2019.

[19] B. Mishra, K. L. Pruseth, P. Hazarika, and S. S. Chinnasamy, "Nature and source of the ore-forming fluids associated with orogenic gold deposits in the Dharwar Craton," Geoscience Frontiers, vol. 9, no. 3, pp. 715-726, 2018.

[20] F. D. Wang, L. I. Yun-Ping, and Y. H. Jia, "Metallogenic regularity and prospecting direction of gold deposits in Qinghai, China," Journal of Earth Sciences \& Environment, vol. 40, no. 2, pp. 162-175, 2018.

[21] G. Song, K. Qin, and G. Li, "Basic characteristics and research progresses of intermediate sulfidation type epithermal gold poly-metallic deposits, and prospects," Acta Petrologica Sinica, vol. 34, no. 3, pp. 748-762, 2018.

[22] A. A. Gasanov, A. V. Naumov, O. V. Yurasova, I. M. Petrov, and T. E. Litvinova, "Certain tendencies in the rare-earth- 
element world market and prospects of Russia," Russian Journal of Non-Ferrous Metals, vol. 59, no. 5, pp. 502-511, 2018.

[23] J. F. Hernández, "machine learning and statistical techniques. An application to the prediction of insolvency in Spanish non-life insurance companies," The International Journal of Digital Accounting Research, vol. 5, no. 9, pp. 1-45, 2020.

[24] Y. Chen, W. Zheng, W. Li, and Y. Huang, "Large group activity security risk assessment and risk early warning based on random forest algorithm," Pattern Recognition Letters, vol. 144, pp. 1-5, 2021.

[25] R. S. Bhadoria and N. S. Chaudhari, "Pragmatic sensory data semantics with service-oriented computing," Journal of Organizational and End User Computing, vol. 31, no. 2, pp. 22$36,2019$.

[26] Q. Wang and P. Lu, "Research on application of artificial intelligence in computer network technology," International Journal of Pattern Recognition and Artificial Intelligence, vol. 33, no. 5, p. 1959015, 2019.

[27] P. Shan, X. Lai, and X. Liu, "Correlational analytical characterization of energy dissipation-liberation and acoustic emission during coal and rock fracture inducing by underground coal excavation," Energies, vol. 12, no. 12, p. 2382, 2019. 